Supporting Information of

\title{
Colloidal Supraballs of Mesoporous Silica Nanoparticles as Bioresorbable Adhesives for Hydrogels
}

Jae Seon Baik ${ }^{+, a}$, Sang A Kim ${ }^{+, a}$, Dae-Woong Jung, Weon-Sik Chae ${ }^{\mathrm{b}}$, Changhyun Pang ${ }^{\mathrm{a}}$, Suk Ho Bhang ${ }^{\mathrm{a}}$, Laurent Cortéb,c, and Gi-Ra Yi*,a,d

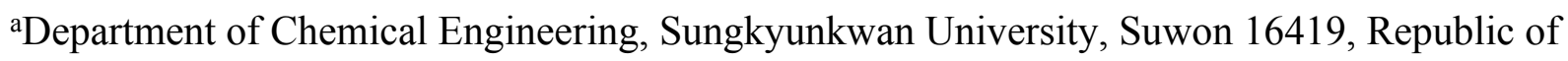
Korea

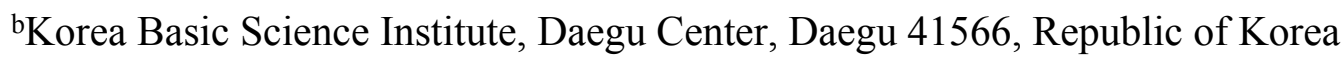

${ }^{\mathrm{c} C h i m i e}$ Moléculaire, Macromoléculaire, et Matériaux, CNRS UMR 7167, ESPCI Paris and

${ }^{\mathrm{d} C e n t r e}$ des Matériaux, CNRS UMR 7633, MINES ParisTech, PSL University, France

eDepartment of Chemical Engineering, Pohang University of Science and Technology

(POSTECH), Pohang, Gyeongbuk 37673, Republic of Korea.

*Corresponding author: E-mail: yigira@postech.ac.kr 


\section{Calculation of the area density of deposited particle coating.}

Coating deposition is characterized by two experimental parameters: $\widetilde{V}_{d}$ the deposited volume per unit area $\left(20 \mu \mathrm{L} / \mathrm{cm}^{2}\right)$ and $C_{m}$ the particle concentration in mass of particles per mass of solution $(\mathrm{wt} \%)$

The area density of the deposited coating (mass of particles deposited per unit area), $\tilde{m}$, can be expressed as a function of $\tilde{V}_{d}$ and $C_{m}$ as follows.

It is given by:

$$
\tilde{m}=C_{v} \times \tilde{V}_{d}
$$

where $C_{v}$ is the particle concentration in mass of particles per unit volume of solution.

By definition, $C_{v}$ and $C_{m}$ are given by:

$$
C_{v}=\frac{m_{\mathrm{SiO}_{2}}}{V_{\mathrm{Total}}}=\frac{m_{\mathrm{SiO}_{2}}}{\frac{m_{\mathrm{SiO}_{2}}}{\rho_{\mathrm{SiO}_{2}}}+\frac{m_{\mathrm{H}_{2} \mathrm{O}}}{\rho_{\mathrm{H}_{2} \mathrm{O}}}} \quad \text { and } \quad C_{m}=\frac{m_{\mathrm{SiO}_{2}}}{m_{\mathrm{SiO}_{2}}+m_{\mathrm{H}_{2} \mathrm{O}}}
$$

where $m_{\mathrm{SiO}_{2}}$ and $m_{\mathrm{H}_{2} \mathrm{O}}$ are the masses of amorphous silica and water, respectively in a given volume of solution, $V_{\text {Total, }}$, and $\rho_{\mathrm{SiO}_{2}}\left(2.196 \mathrm{~g} / \mathrm{cm}^{3}\right)$ and $\rho_{\mathrm{H}_{2} \mathrm{O}}\left(1 \mathrm{~g} / \mathrm{cm}^{3}\right)$ are the densities of amorphous silica and water, respectively.

Therefore, $C_{v}$ is related to $C_{m}$ by:

$$
C_{v}=\frac{1}{\frac{1}{\rho_{\mathrm{SiO}_{2}}}+\frac{1-C_{m} 1}{C_{m} \rho_{\mathrm{H}_{2} \mathrm{O}}}}
$$

As a result,

$$
\tilde{m}=\frac{\tilde{V}_{d}}{\frac{1}{\rho_{\mathrm{H}_{2} \mathrm{O}} \cdot C_{m}}-\left(\frac{1}{\rho_{\mathrm{H}_{2} \mathrm{O}}}-\frac{1}{\rho_{\mathrm{SiO}_{2}}}\right)}
$$




\section{Calculation of the number of particle layers and the mean coating thickness.}

From the area density of the deposited coating, $\tilde{m}$, one can estimate the mean number of nanoparticles or supraballs per unit area, $\tilde{N}$, as follows:

$$
\tilde{N}=\tilde{m} / m_{p a r t}
$$

where $m_{\text {part }}$ is the mass of one particle (nanoparticle or supraball).

$m_{\text {part }}$ is given by:

$$
m_{\text {part }}=\frac{\pi}{6} d_{\text {part }}^{3} \rho_{\text {part }}
$$

where $d_{\text {part }}$ and $\rho_{\text {part }}$ are the diameter and density of one particle, respectively.

$\rho_{\text {part }}$ is given by:

$$
\rho_{\text {part }}=\frac{\rho_{\mathrm{SiO}_{2}}}{1+\rho_{\mathrm{SiO}_{2}} \cdot v_{S p}}
$$

where $v_{S p}$ is the specific pore volume of one particle (as measured by BET) and $\rho_{\mathrm{SiO}_{2}}\left(2.196 \mathrm{~g} / \mathrm{cm}^{3}\right.$ ) and $\rho_{\mathrm{H}_{2} \mathrm{O}}\left(1 \mathrm{~g} / \mathrm{cm}^{3}\right)$ are the densities of amorphous silica and water, respectively.

As a result,

$$
\tilde{N}=\frac{6 \tilde{m}\left(1+\rho_{\mathrm{SiO}_{2}} \cdot v_{s p}\right)}{\pi_{d_{\text {part }}{ }^{3}} \rho_{\mathrm{SiO}_{2}}}
$$

Assuming a hexagonal packing of the particles, the maximum number of particles per unit area contained in one flat monolayer is given by:

$$
\tilde{N}_{\text {monolayer }}=0.9069 \cdot \frac{4}{\pi d_{\text {part }}^{2}}
$$


For a $10 \mathrm{~mm} \times 5 \mathrm{~mm}$ surface of hydrogel, this formula gives that $1.60 \times 10^{10}$ of $\mathrm{SN}$ and $1.36 \times 10^{10}$ of MSN could be stack up in single layer. In case of SB-SN, SB-MSN, and MSB-MSN, the maximum number of particles in single layer is calculated as $4.71 \times 10^{6}, 3.61 \times 10^{6}$, and $5.30 \times 10^{6}$ per $50 \mathrm{~mm}^{2}$, respectively.

The number of particle layers composing the coatings can then be approximated by:

$$
\begin{gathered}
n_{\text {layer }}=\tilde{N} / \tilde{N}_{\text {monolayer }} \\
n_{\text {layer }}=1.65 \cdot \frac{\tilde{m}\left(1+\rho_{\mathrm{SiO}_{2}} \cdot v_{\text {sp }}\right)}{d_{\text {part }} \quad \rho_{\mathrm{SiO}_{2}}}
\end{gathered}
$$

The mean coating thickness is then given by:

$$
\begin{gathered}
\text { Coating thickness }=n_{\text {layer }} \cdot d_{\text {part }} \\
\text { Coating thickness }=1.65 \cdot \tilde{m} \cdot \frac{\left(1+\rho_{S_{i O_{2}}} \cdot v_{s p}\right)}{\rho_{S_{i O}}}
\end{gathered}
$$

This calculation was used in Figure S4 showing the adhesion energy as a function of the mean coating thickness. We also calculated the number of layers for each concentration solution with this method as shown in Figure S5a.

The number of nanoparticle layers in one supraball (n) was calculated by dividing the supraball diameter $\left(d_{\text {supraball }}\right)$ with diameter of particles $\left(d_{\text {nanoparticle }}\right)$. With this calculation, the number of nanoparticle layers in one supraball were 33, 31, and 25.5 in 3.5- $\mu \mathrm{m}$ SB-SN, 4.0- $\mu \mathrm{m}$ SB-MSN and 3.3- $\mu \mathrm{m}$ MSB-MSN, respectively. In other words, when a monolayer of SB-MSN and MSB-MSN $(1 \mathrm{wt} \%)$ corresponds to 61 or $50(=2 \mathrm{n}-1)$ layers of mesoporous silica nanoparticles. 


\section{Calculation of the specific outer surface area available for polymer adsorption}

We define the specific outer surface area, $a_{T O}$, as the total surface area in a coating that is available for the adsorption of hydrogel strands.

\subsection{Case of unaggregated nanoparticle coating:}

In the case of unaggregated nanoparticles (SN, MSN), we use a method similar to the one described in previous work. ${ }^{[12]}$

For non-porous nanoparticles (SN), hydrogel chains can adsorb on all the outer surface of the nanoparticles and $a_{T O}$ is simply given by:

$$
\begin{gathered}
a_{T O}=\frac{\tilde{m}}{\rho_{S N} \cdot V_{S N}} \cdot S_{S N} \\
a_{T O}=\frac{6 \tilde{m}}{\rho_{S i O_{2}} \cdot d_{S N}}
\end{gathered}
$$

where $\rho_{S N}\left(=\rho_{S i O_{2}}\right), V_{S N}, S_{S N}$ and $d_{S N}$ are the density, volume, surface area and diameter of a single SN nanoparticle, respectively.

For mesoporous nanoparticles (MSN), hydrogel chains cannot reach the deep interior structure of the nanoparticles because of the entropic cost of stretching those chains inside the mesopores. On each nanoparticle, we assume that polymer chains can only adsorb at a depth $\xi$ inside cylindrical mesopores having a diameter $d_{\text {pore }}$ exposed at the nanoparticle surface. Following the same approach as for SN particles, the expression of $a_{T O}$ is thus given by:

$$
a_{T O}=\frac{\tilde{m}}{\rho_{M S N} \cdot V_{M S N}} \cdot\left(\pi d_{M S N}^{2}+n \pi \xi d_{\text {pore }}\right)
$$


where $\rho_{M S N}, V_{M S N}, S_{M S N}$ and $d_{M S N}$ are the density, volume, surface area and diameter of a single MSN nanoparticle, respectively and $n(=159)$ is the number of pores at the surface of one MSN nanoparticle. ${ }^{[12]}$

The density of one MSN nanoparticle can be expressed as:

$$
\rho_{M S N}=\frac{\rho_{\mathrm{SiO}_{2}}}{1+\rho_{\mathrm{SiO}_{2}} \cdot v_{S p_{-} M S N}}
$$

where $v_{S p_{-} M S N}$ is the specific pore volume of MSN particles (as measured by BET).

As a result, the value of $a_{T O}$ can be calculated using the following expression:

$$
a_{T O}=\frac{6 \tilde{m}}{\rho_{S i O_{2}} \cdot d_{M S N}}\left(1+\rho_{S i O_{2}} \cdot v_{S p_{-} M S N}\right)\left(1+n \frac{\xi d_{\text {pore }}}{d_{M S N}^{2}}\right)
$$

For instance, considering non-porous silica nanoparticle (SN) with a diameter of $60 \mathrm{~nm}$ and mesoporous silica nanoparticle (MSN) with a diameter of $65 \mathrm{~nm}$ and a pore diameter of $7.2 \mathrm{~nm}$, we find that for a same concentration of deposited solution, penetration of polymer chains at a depth $\xi=d_{\text {pore }} / 2$ gives a specific outer surface area of MSN that is about 5 times larger than that of SN.

\subsection{Case of supraballs:}

In the case of supraballs (SB-SN, SB-MSN), the entropic cost of deformation of the network chains to diffuse between the sintered nanoparticles and the reduced connectivity of the interstices are expected to strongly hinder the amount of surface available for polymer adsorption. Therefore, we assume that polymer strands probably only penetrate within a depth $h$ in an outer shell of the 
supraballs. Assuming $h$ is much smaller than the supraball diameter $D_{S B}$, the volume of the supraball accessible for polymer adsorption is then given by:

$$
V_{S B_{-} \text {Shell }}=\frac{\pi D_{S B}{ }^{3}}{6}-\frac{\pi\left(D_{S B}-2 h\right)^{3}}{6} \approx \pi D_{S B}{ }^{2} h
$$

The number of nanoparticles contained in this outer shell is given approximately by:

$$
n_{N P \_ \text {in_the } \text { shell }}=\frac{V_{S B_{-} \text {Shell }}}{\pi d^{3} / 6} \approx \frac{6 D_{S B}{ }^{2} h}{d^{3}}
$$

where $d$ is the diameter of one nanoparticle in the supraball.

As a result, the specific outer surface area, $a_{T O}$, for a supraball coating can be estimated by:

$$
a_{T O}=\frac{\tilde{m}}{\rho_{S B} \cdot V_{S B}} \cdot \frac{6 D_{S B}^{2} h}{d^{3}} \cdot S
$$

where $\rho_{S B}$ and $V_{S B}$ are the density and volume of a single supraball, respectively, and $S$ is the surface available for polymer adsorption on one nanoparticle as calculated above: for SN, $S=\pi d_{S N}^{2}$ and for MSN, $S=\left(\pi d_{M S N}^{2}+n \pi \xi d_{\text {pore }}\right)$.

We find the following expressions for $a_{T O}$ :

- For SB-SN:

$$
\begin{gathered}
a_{T O}=\frac{\tilde{m}}{\rho_{S B-S N} \cdot V_{S B-S N}} \cdot \frac{6 D_{S B-S N}{ }^{2} h}{d_{S N}{ }^{3}} \cdot \pi d_{S N}{ }^{2} \\
a_{T O}=\frac{36 \tilde{m}}{\rho_{S i O_{2}} \cdot D_{S B-S N}} \cdot \frac{h}{d_{S N}} \cdot\left(1+\rho_{S i O_{2}} \cdot v_{S p_{-} S B-S N}\right)
\end{gathered}
$$

- For SB-MSN:

$$
a_{T O}=\frac{\tilde{m}}{\rho_{S B-M S N} \cdot V_{S B-M S N}} \cdot \frac{6 D_{S B-M S N}{ }^{2} h}{d_{M S N}{ }^{3}} \cdot\left(\pi d_{M S N}{ }^{2}+n \pi \xi d_{\text {pore }}\right)
$$




$$
a_{T O}=\frac{36 \tilde{m}}{\rho_{S i O_{2}} \cdot D_{S B-M S N}} \cdot \frac{h}{d_{M S N}} \cdot\left(1+\rho_{S_{i O}} \cdot v_{S p \_S B-M S N}\right) \cdot\left(1+n \frac{\xi d_{\text {pore }}}{d_{M S N}{ }^{2}}\right)
$$

- For MSB-MSN: For macroporous supraballs of MSN nanoparticles, an additional correction factor is needed to account for the increase in supraball surface caused by the macropores, as follows:

$$
\begin{gathered}
a_{T O}=\frac{36 \tilde{m}}{\rho_{S i O_{2}} \cdot} D_{M S B-M S N} \cdot \frac{h}{d_{M S N}} \cdot\left(1+\rho_{S_{i O}} \cdot v_{S p_{-} M S B-M S N}\right) \cdot\left(1+n \frac{\xi d_{\text {pore }}}{d_{M S N}^{2}}\right) \\
\cdot\left(1+\frac{n_{\text {macropore }}}{2}\left(\frac{r_{\text {macropore }}}{D_{M S B-M S N}}\right)^{2}\right)
\end{gathered}
$$

where $n_{\text {macropore }}$ and $r_{\text {macropore }}$ are the number and radius of the macropores exposed at the surface of one MSB-MSN supraball, respectively. 
Table S1. Experimental condition for preparing colloidal supraballs via spray drying.

\begin{tabular}{cccccccc}
\hline & $\begin{array}{c}\text { Primary } \\
\text { particle } \\
(\text { Template })\end{array}$ & $\begin{array}{c}\text { Conc. of } \\
\text { nanoparticles } \\
{[\mathrm{wt} \%]}\end{array}$ & $\begin{array}{c}\text { Inlet } \\
\text { temp. } \\
{\left[{ }^{\circ} \mathrm{C}\right]}\end{array}$ & $\begin{array}{c}\text { Outlet } \\
\text { temp. } \\
{\left[{ }^{\circ} \mathrm{C}\right]}\end{array}$ & $\begin{array}{c}\text { Blower } \\
\text { Flow rate } \\
{\left[\mathrm{m}^{3} / \mathrm{min}\right]}\end{array}$ & $\begin{array}{c}\text { Atomizing } \\
\text { Pressure } \\
{[\times 10 \mathrm{kPa}]}\end{array}$ & Speed \\
\hline SB-SN & SN & 6 & 125 & 85 & 0.3 & 7 & 2.5 \\
SB-MSN & MSN & 2 & 145 & 90 & 0.5 & 9 & 3.5 \\
MSB- & MSN & 6 & 145 & 90 & 0.5 & 5 & 2.5 \\
MSN & (PS) & $(2.5)$ & & & & & \\
\hline
\end{tabular}



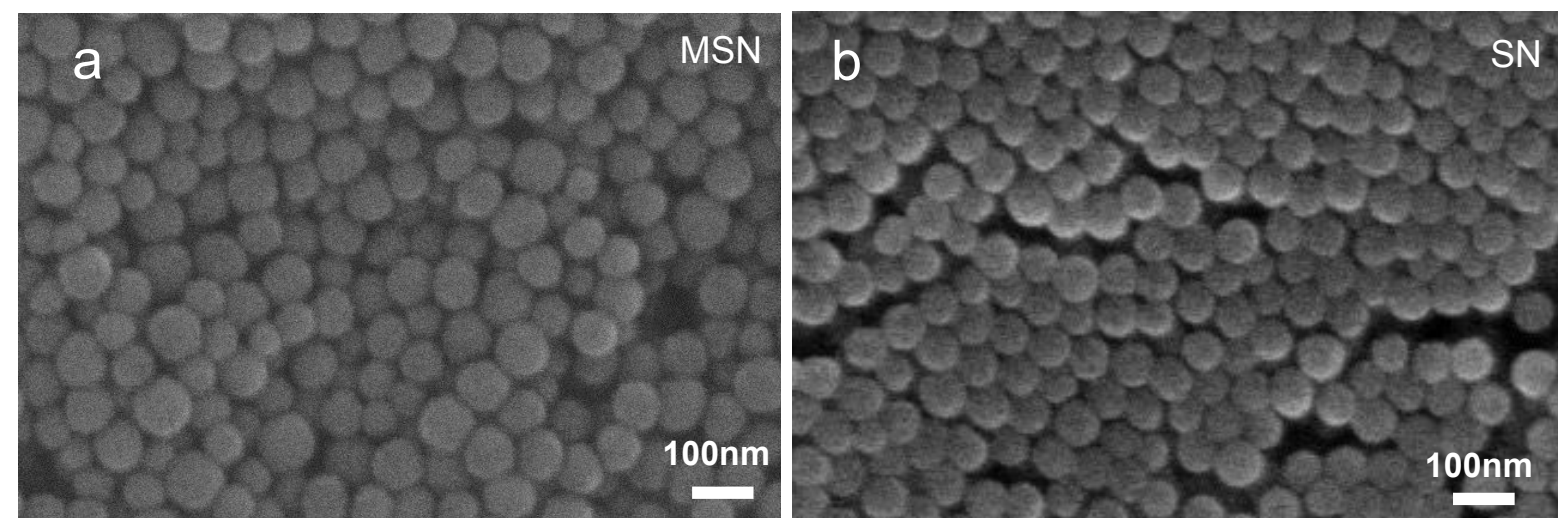

Figure S1. SEM images of (a) mesoporous silica nanoparticles (MSN) and (b) non-porous silica nanoparticles $(\mathrm{SN})$. 


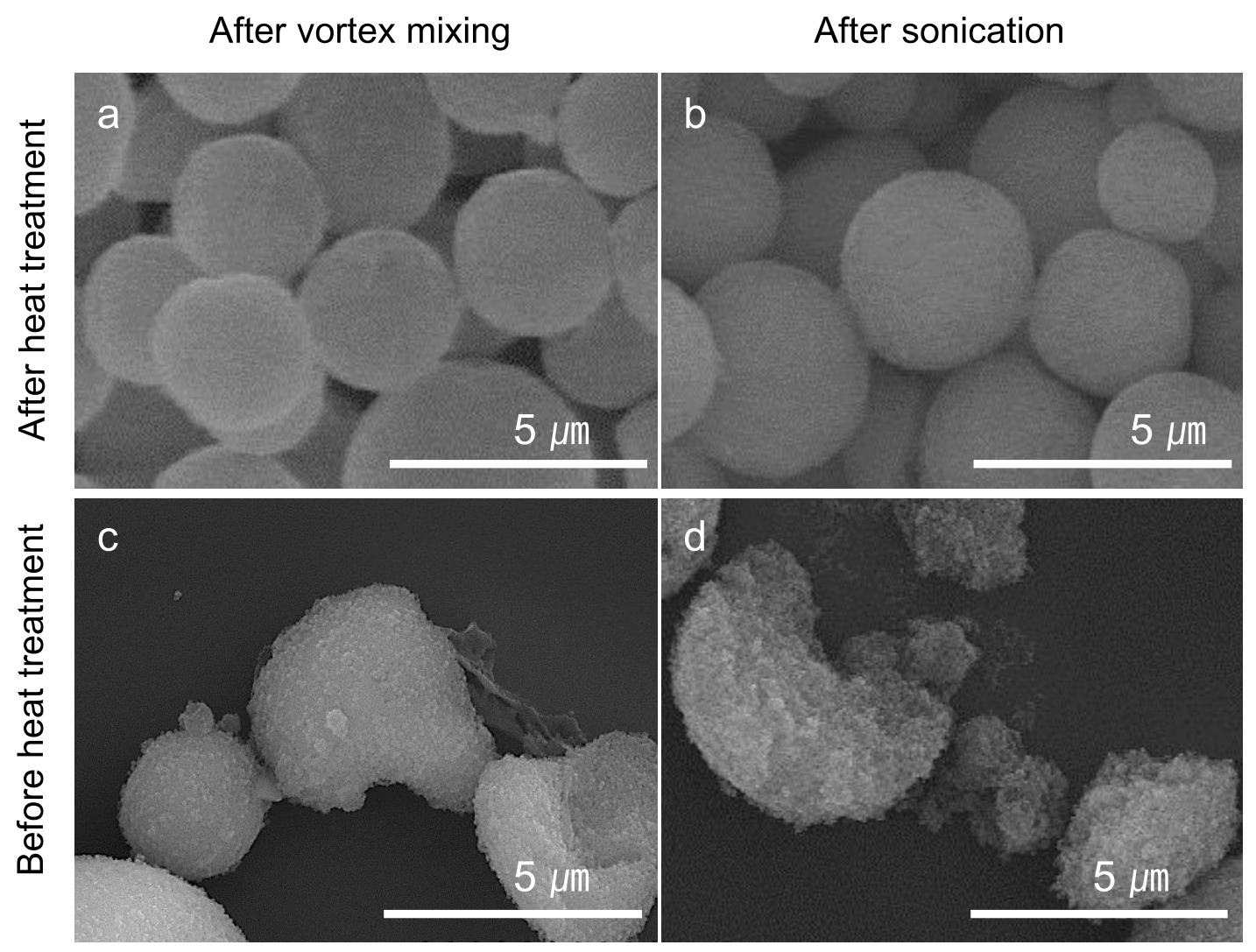

Figure S2. SEM images of supraballs of mesoporous silica nanoparticles (SB-MSN) in water under external forces such as $(\mathrm{a}, \mathrm{c})$ vortex and $(\mathrm{b}, \mathrm{d})$ sonication $(\mathrm{a}, \mathrm{b})$ after and $(\mathrm{c}, \mathrm{d})$ before heat treatment.
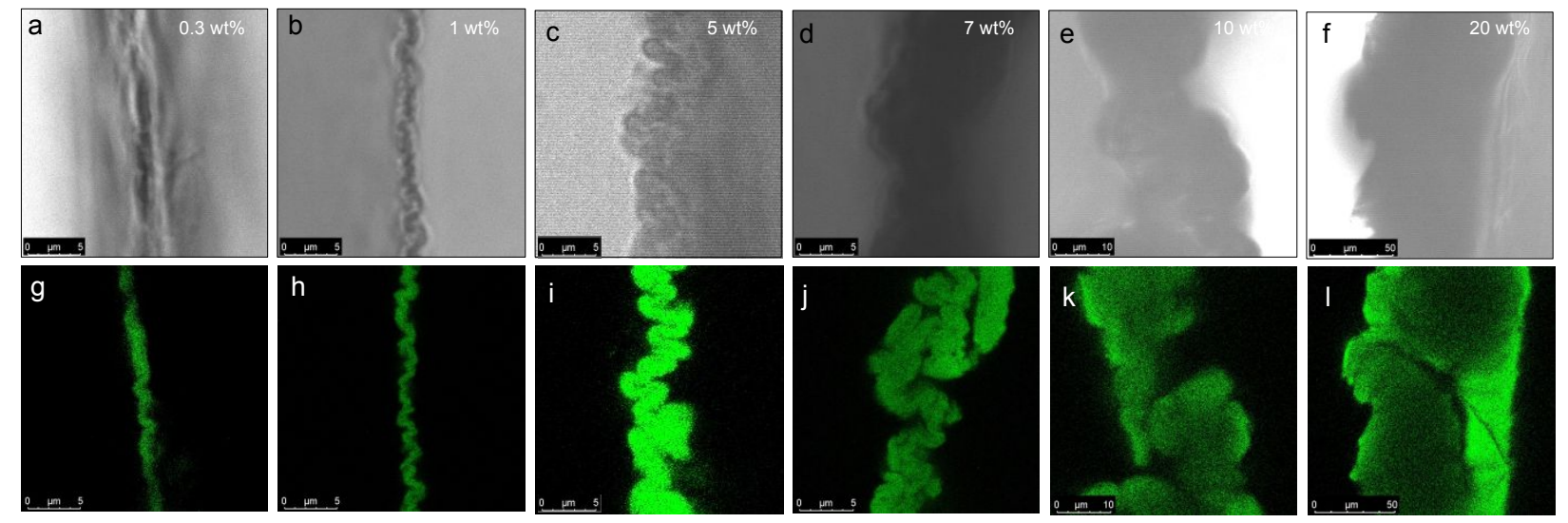

Figure S3. (a-f) Bright-field and (g-l) fluorescent confocal microscopic images of hydrogelhydrogel interface glued with (a,g) 0.3, (b,h) 1, (c,i) 5, (d,j) 7, (e,k) 10 and (f,l) 20-wt.\% solutions of FITC-labelled MSN. 


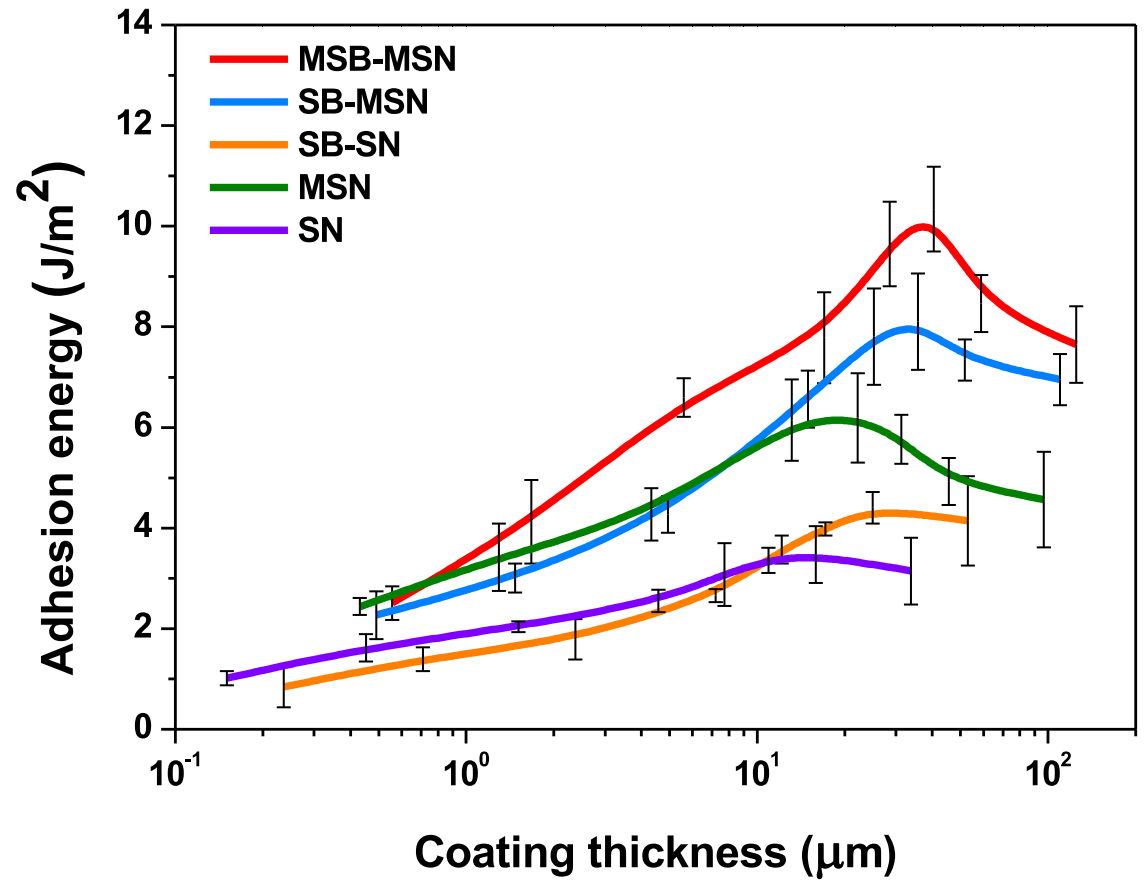

Figure S4. Adhesion energy as a function of the average thickness of the deposited particle coating. 


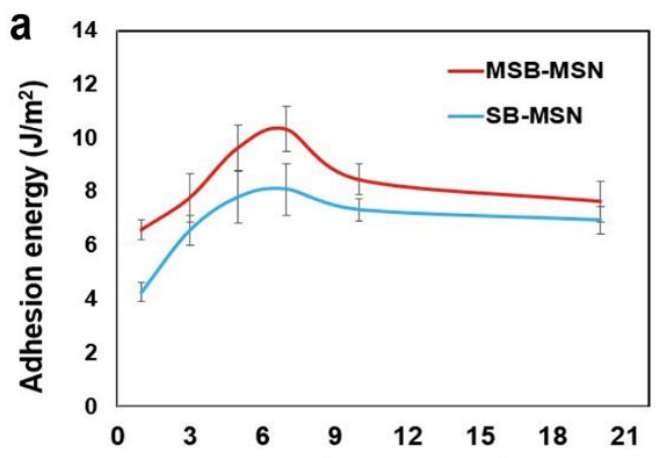

b The number of the layer of supraballs

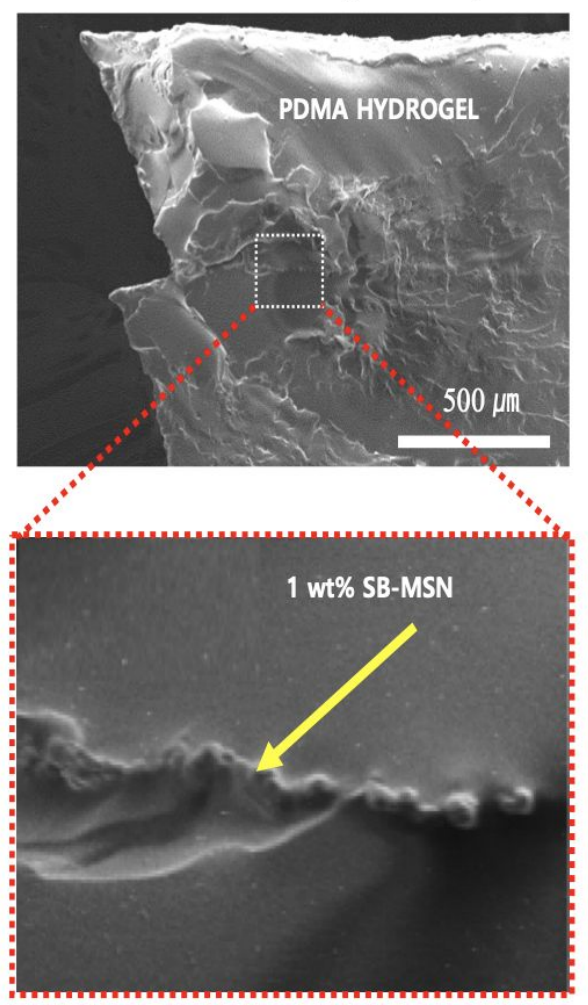

Figure S5. Adhesion energy as a function of the (a) layers of supraballs and (b) cross-sectional SEM images of two hydrogel films glued with $1 \mathrm{wt} \%$ SB-MSN. 


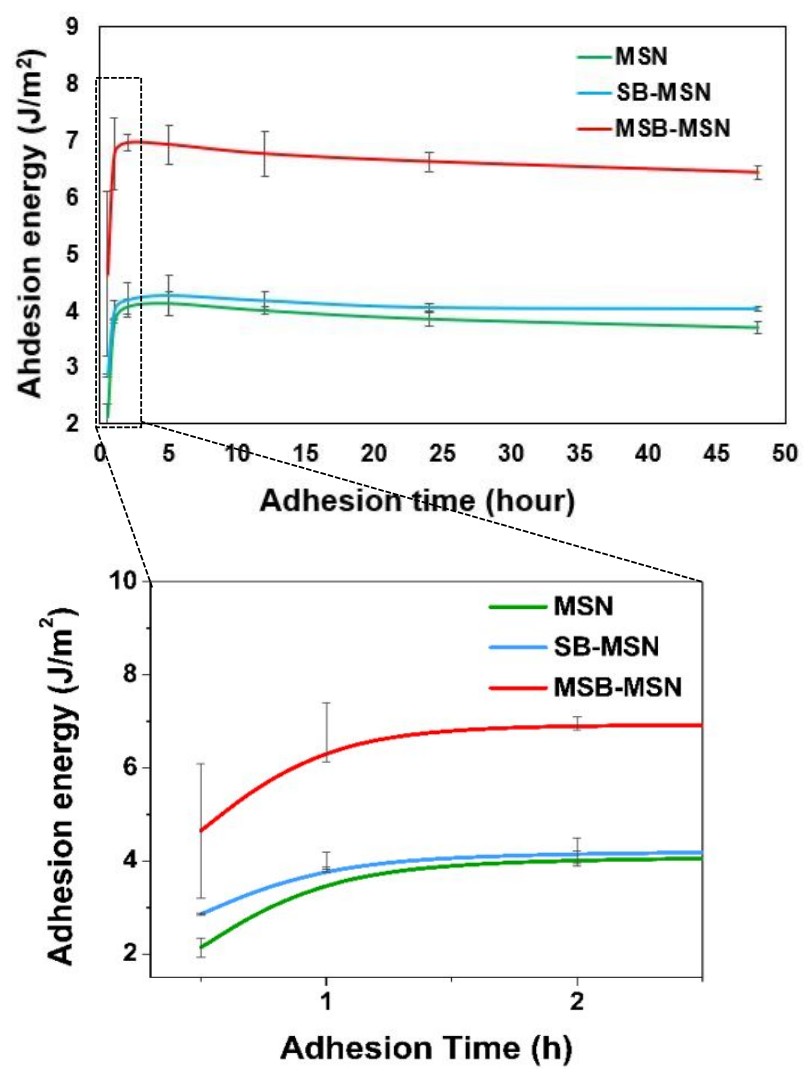

Figure S6. Adhesion energy between hydrogel with 1-wt $\%$ MSN, SB-MSN, and MSB-MSN as a function of the duration time.

\section{References}

[S1] W. Stöber, A. Fink, E. Bohn, J. Colloid Interface Sci. 1968, 26, 62.

[S2] J. Lee, C. K. Hong, S. Choe, S. E. Shim, J. Colloid Interface Sci. 2007, 310, 112.

[S3] H. I. Hussain, Z. Yi, J. E. Rookes, L. X. Kong, D. M. Cahill, J. Nanoparticle Res. 2013, 15, 1676, 3 